\title{
The natural history of aneurysmal coronary artery disease
}

Vassilios P Demopoulos, Christopher D Olympios, Constantine N Fakiolas, Evagelos G Pissimissis, Nicholas M Economides, Evdokia Adamopoulou, Stefanos G Foussas, Dennis V Cokkinos

\begin{abstract}
Objective-To assess the contribution of coronary artery ectasia, either isolated or in association with obstructive coronary artery disease, to morbidity and mortality from ischaemic heart disease.

Design-A retrospective study of patients undergoing coronary arteriography at a tertiary cardiac centre.

Patients and methods-The epidemiological, clinical, arteriographic, and follow up characteristics of three groups of patients were examined: group A, 172 patients with coronary artery ectasia and coexisting significant coronary artery disease; group B, 31 patients with coronary artery ectasia only; group C, 165 patients with significant coronary artery disease but without ectasia, matched for sex and age with group $\mathbf{A}$.
\end{abstract}

Results-Group A patients had a similar incidence of a previous myocardial infarction to group $C$ patients $(61.6 \% v$ $64 \cdot 2 \%$ ), exercise performance, severity of obstructive lesions (CASS score $2 \cdot 19 v$ $2 \cdot 14)$, and similar distribution of diseased vessels. At follow up of approximately two years they experienced a similar incidence of unstable angina $(7.5 \% v \quad 4.4 \%)$ and myocardial infarction plus cardiac death $(4.9 \% \quad v 6 \cdot 1 \%)$. They underwent bypass surgery with similar frequency $(39 \% v 42 \%)$ but there was a lower frequency of percutaneous transluminal coronary angioplasty $(5.8 \% \quad v \quad 17 \%$, $\mathbf{P}<0 \cdot 01)$. Patients with pure coronary ectasia (group B) had a lower incidence of a previous myocardial infarction $(38.7 \%$, $12 / 31, P<0.05)$ than the two other groups. The infarct in all cases was related to an ectatic artery. Their exercise performance and ejection fraction (9 (SD 3) minutes and 56.5(9)\%) were higher $(P<$ 0.01) than group A (5 (2) minutes, $48 \cdot 3(10) \%)$ and group C (5.3 (2) minutes, $49 \cdot 3(10) \%$ ). Group B had no myocardial infarctions, cardiac death, surgery, or intervention at follow up; $4.4 \%$ (5/115) developed unstable angina. The incidence of angina at study entry was similar in all three groups (38.7-49.7\%).

Conclusions-Coronary artery ectasia does not confer added risk in patients with coexisting obstructive coronary artery disease. Although there is a measurable incidence of previous myocardial infarction, patients with pure ectasia have a good prognosis. The wisdom of giving oral anticoagulants to such patients is questioned.

(Heart 1997;78:136-141)

Keywords: coronary artery; ectasia; aneurysms; coronary artery disease

Coronary artery ectasia or aneurysmal coronary artery disease is defined as distension of a part of a coronary vessel of up to one and a half times the diameter of an adjacent normal segment. ${ }^{1}$ This pathological picture can be diffuse, affecting the entire length of a coronary artery, but it can also be discrete or localised. When the dilatation involves the whole vessel it is more appropriate to use the word "ectasia" than aneurysmal disease..$^{2-4}$

Coronary artery ectasia is considered an accompaniment or a variant of coronary atherosclerosis. ${ }^{1}$ It has been suggested that the presence of the aneurysmal segments produces sluggish or turbulent blood flow, with an increased incidence of ischaemic manifestations or occurrence of acute myocardial infarction as a result, regardless of the actual severity of the coexisting stenotic coronary lesions. ${ }^{25}$ It has been postulated that patients with coronary ectasia but without significant coexistent coronary artery disease have a higher incidence of adverse events than individuals with normal coronary arteries. ${ }^{2}$ The best treatment has not been clearly defined.

Although this entity has been the subject of many studies, a complete clinical picture has not emerged. We thus decided to investigate the clinical characteristics of the condition, to differentiate between ectasia with and without coexistent obstructive coronary artery disease, and to compare affected individuals with a group of patients with coronary artery disease of similar severity but no ectasia. We also tried to investigate the coexistence of ectasia and stenotic lesions in the same vascular segments, and to differentiate these from stenotic lesions in segments without aneurysmal lesions.

\section{Methods}

We retrospectively studied 3900 patients who had been referred for coronary arteriography. The definition of "aneurysmal coronary artery disease" was that employed by the coronary artery surgery study (CASS). ${ }^{1}$ According to CASS, a vessel is considered to be aneurysmal 
if its diameter is 1.5 times greater than that of the adjacent normal segment in segmental ectasia, or the largest diameter of any normal segment of the patient's coronary arterial tree in diffuse ectasia.

Three groups of patients were studied:

1 Group A consisted of 172 patients who were judged to have coronary artery ectasia with coexisting coronary artery disease defined as $>70 \%$ diameter stenosis of any of the major coronary arteries, or $\geqslant 50 \%$ stenosis of the left main trunk. ${ }^{6}$

2 Group B consisted of 31 patients who had aneurysmal disease but no significant coronary stenosis $(<50 \%$ diameter stenosis of any vessel).

3 Group C consisted of 165 patients with significant coronary artery disease but without any evidence of ectasia, matched for sex and age with the patients in group $A$. These patients were consecutively included from our coronary arteriography list in parallel with the recruitment of the patients in groups $A$ and $B$. The criterion for inclusion was the presence of any significant disease. The matched patients were selected over the same time interval as the patients with coronary ectasia.

The reason for catheterisation in the patients of all groups was either typical angina or chest pain suggestive of angina, a previous myocardial infarction, or a positive exercise test.

Calculations of vessel lumen diameter to assess the severity of the coronary lesion were visually graded by two experienced angiographers, working independently. A vessel diameter stenosis of $>70 \%$ ( $>50 \%$ for the main stem) was considered significant, according to the criteria employed by CASS $^{1}$ and Aintablian et $a l,{ }^{7}$ when it was situated in a nonectatic segment of the affected vessel. In the cases where the stenosis was situated in an ectatic segment, the same criterion was applied. The degree of stenosis was evaluated by comparison to the proximal and distal ectatic segments. The ejection fraction was calculated by the Sandler and Dodge single plane projection (right anterior oblique) method. ${ }^{8}$

Patients with valvar (including mitral valve prolapse) or congenital heart disease, cardiomyopathy, left ventricular hypertrophy by echocardiography, or who had previously been subjected to myocardial revascularisation were excluded.

In groups A and B with coronary ectasia, the subgroup classification used by Markis et $a l^{2}$ was employed. This describes four types of ectasia:

I. Diffuse ectasia with aneurysmal lesions in two vessels.

II. Diffuse ectasia in one vessel and discrete in another.

III. Diffuse ectasia in one vessel.

IV. Discrete ectasia in one vessel.

The epidemiological and clinical data as regards risk factors, incidence of a previous $Q$ wave or non- $Q$ wave myocardial infarction documented at hospital admission, and angina pectoris incidence were compared in the three groups. Hypertension was defined as blood pressure $>140 / 90 \mathrm{~mm} \mathrm{Hg}$, and hyperlipidaemia as total cholesterol $>200 \mathrm{mg} / \mathrm{dl}$. Exercise testing was performed according to the Bruce protocol. It was considered to be positive when a horizontal or downsloping ST segment depression of $>2 \mathrm{~mm}$ during or $>1 \mathrm{~mm}$ after exercise was recorded, measured at $80 \mathrm{~ms}$ from the $\mathrm{J}$ point, with or without anginal pain. Coronary artery disease severity (according to the criteria employed by the CASS study ${ }^{1}$ ) and the development of collateral vessels (according to the criteria employed by Cohen and Rentrop ${ }^{9}$ ) were also compared in groups $A$ and $C$.

Follow up data on these three groups of patients over two years were retrospectively evaluated. The following characteristics were noted: (1) type of treatment, according to the initial advice given by a panel of physicians not involved in the study and subsequently modified by their personal physicians-specifically, the continuation of medical treatment or the performance of percutaneous transluminal coronary angioplasty or aortocoronary bypass treatment was noted; (2) hospital admission for unstable angina or acute myocardial infarction, and the occurrence of cardiac death.

\section{STATISTICAL ANALYSIS}

Comparison of the incidence of the various data among the three groups was carried out by the $\chi^{2}$ test.

\section{Results}

\section{INCIDENCE}

Among the 3900 coronary arteriograms evaluated, 203 patients were judged to have coronary ectasia, an incidence of $5.3 \%$. From these, 172 patients (group A) were judged to have significant coexistent coronary artery disease, while 31 had otherwise nearly normal coronary vessels (group B).

\section{EPIDEMIOLOGICAL FINDINGS}

In the two groups with coronary artery ectasia, males were equally affected. Sex and age were matched in groups $\mathrm{A}$ and $\mathrm{C}$. There was no difference in the three groups with regard to history of hyperlipidaemia, hypertension, and diabetes mellitus. Smoking was more common in groups $\mathrm{A}$ and $\mathrm{C}$ with coronary artery disease. All three groups had a similar incidence of angina; however, group B patients had a significantly lower incidence $(P<0.05)$ of previous $Q$ wave or non- $Q$ wave myocardial infarction than patients in groups $A$ and $C$ (table 1).

\section{EXERCISE TESTING}

Exercise tolerance was similar in group A (total exercise time $5 \cdot 1$ (SD 2) minutes) and group C (5.3 (2) minutes), but was significantly longer in group B $(9 \cdot 2(3), P<0.001)$. However, the incidence of a positive test was similar in the three groups: $85.6 \%(85 / 99)$ in group $\mathrm{A}, 66.7 \%(8 / 12)$ in group $\mathrm{B}$, and $80 \%$ $(68 / 85)$ in group $C$, by the $\chi^{2}$ test. Although the incidence of a positive test was lower in 
Table 1 Epidemiological findings in the three study groups

\begin{tabular}{|c|c|c|c|}
\hline & Group $A$ & Group $B$ & Group $C$ \\
\hline $\begin{array}{l}\text { Number } \\
\text { Male sex \% (n) } \\
\text { Age (years) } \\
\text { Smoking \% (n) } \\
\text { Hyperlipidaemia \% (n) } \\
\text { Diabetes mellitus \% (n) } \\
\text { Hypertension \% (n) } \\
\text { Family history of CAD events \% (n) } \\
\text { Angina \% (n) } \\
\text { Previous myocardial infarction \% (n) }\end{array}$ & $\begin{array}{l}172 \\
93 \cdot 6(161) \\
57 \cdot 3(10) \\
74 \cdot 4(128)^{\star} \\
50 \cdot 6(87) \\
16 \cdot 3(28) \\
32 \cdot 5(56) \\
33 \cdot 7(58) \\
41 \cdot 3(71) \\
61 \cdot 6(106) \ddagger\end{array}$ & $\begin{array}{l}31 \\
87(27) \\
54 \cdot 8(13) \dagger \\
41 \cdot 9(13) \dagger \\
35 \cdot 5(11) \\
12 \cdot 9(4) \\
35 \cdot 5(11) \\
25(8) \\
38 \cdot 7(12) \\
38 \cdot 7(12) \dagger\end{array}$ & $\begin{array}{l}165 \\
87 \cdot 3(144) \\
57 \cdot 6(10) \\
71(117) \\
46(76) \\
20(33) \\
35 \cdot 1(58) \\
24 \cdot 2(40) \\
49 \cdot 7(82) \\
64 \cdot 2(106)\end{array}$ \\
\hline
\end{tabular}

$\star \mathrm{P}<0.001 v$ group $\mathrm{B} ; \nmid \mathrm{P}<0.001 v$ group $\mathrm{C} ; \ddagger \mathrm{P}<0.05 v$ group $\mathrm{B}$

Group A, patients with coronary ectasia and coexisting coronary artery disease (CAD); Group $B$ patients with coronary artery ectasia without significant coexisting $\mathrm{CAD}$; Group C, patients with significant CAD without ectasia.

Figure 1 Severity of coronary artery disease in relation to the number of vessels involved.

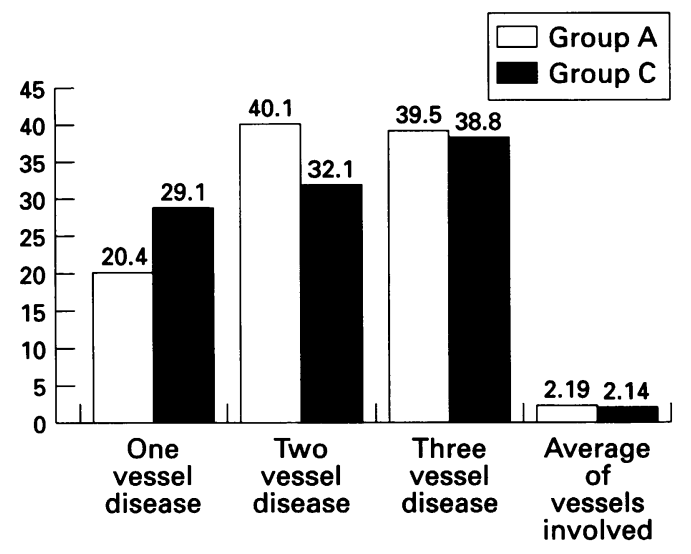

group B this did not reach statistical significance.

CORONARY ANGIOGRAPHIC PICTURE

By the Markis criteria ${ }^{2}$ the majority of group A patients $(58.8 \% ; 101 / 172)$ had mainly type III ectasia, with a similar incidence of the other three types: type I, $14 \%$ (24/172); type II, $11.6 \%$ (20/172); type IV, 15.6\% (27/172). Altogether, involvement of more than one vessel by the aneurysmal process, either diffuse or discrete, was seen in $25.6 \%$ of patients $(44 / 172)$. The right coronary artery was the vessel most frequently involved by the ectatic process $(52.6 \% ; 119 / 226)$, while the circumflex was affected in $24 \cdot 8 \%(56 / 226)$, the left anterior descending in $16.4 \%(37 / 226)$, and the main trunk in $6 \cdot 2 \%(14 / 226)$.

In group $B$ there was a different picture of vessel involvement by the ectatic process. The majority of patients $(45.2 \% ; 14 / 31)$ presented with type I ectasia $(\mathrm{P}<0.001 v$ group $\mathrm{A})$, while $12 \cdot 9 \%$ (4/31) had type II, $32 \cdot 2 \%(10 / 31)$ had type III ( $P<0.01 v$ group $A)$, and $9.7 \%$ (3/31) had type IV. Thus altogether $58 \cdot 1 \%$ of patients $(18 / 31)$ had involvement of more than one vessel by the aneurysmal process $(\mathrm{P}<$ $0.001 v$ group A). The vessel most frequently involved in group B was the right coronary artery in $44.5 \%(16 / 36)$, followed by the left circumflex in $36 \%(13 / 36)$, and the left anterior descending in $16.7 \%(6 / 36)$, while the main stem was involved in $2 \cdot 8 \%(1 / 36)$. These values are not significantly different from group A.

Total coronary artery disease severity, expressed as the number of coronary stenotic lesions per patient, was equivalent in group $\mathrm{A}$ and group C: group A, $2 \cdot 19(0 \cdot 6)$ stenotic vessels, group C, $2 \cdot 14(0 \cdot 8)$ stenotic vessels. The two groups had a similar incidence of one vessel, two vessel, and three vessel disease (fig 1). The precise involvement of the vessels in group $A$ is shown in fig 2 . Analysis of vessel involvement showed that the two groups were similar: in group $\mathrm{A}$, the left main coronary artery was involved in $2.4 \%(9 / 379)$, the left anterior descending in $38.2 \%(145 / 379)$, the left circumflex in $31.4 \%(119 / 379)$, and the right coronary in $28.0 \%(106 / 379)$. The corresponding values for group $\mathrm{C}$ were: left main $3 \cdot 7 \%(13 / 353)$, left anterior descending $37 \cdot 4 \%$ (132/353), left circumflex $29 \cdot 7 \%$ (105/353), and right coronary $29.2 \%(103 / 353)$ (all not significant). In group $\mathrm{A}, 34 \cdot 3 \%$ of the stenotic lesions were in the vessels affected by the ectatic process, while $65.7 \%$ (249) were in the non-ectatic vessels (fig 2). The same figure shows that in the 249 non-ectatic stenotic vessels, the left main coronary artery was affected in $3.6 \%$, the left anterior descending in $51.8 \%$, the circumflex in $30.9 \%$, and the right coronary in $13.7 \%$. When the stenosis was located in the ectatic vessel, but in the non-ectatic segment, there was a similar involvement. However, when the stenosis was located in the
Figure 2 Precise involvement of vessels in group $A$ (patients with ectasia and coronary artery disease). $L A D$, left anterior descending artery; LCX, left circumflex artery; $L M$, left main artery; $R C A$, right coronary artery. ${ }^{\star} P<0.001$ for $L A D$ and $R C A$ involvement for subgroup $1 a$ versus $I b$ and II; and $P<0.001$ for group I versus II.

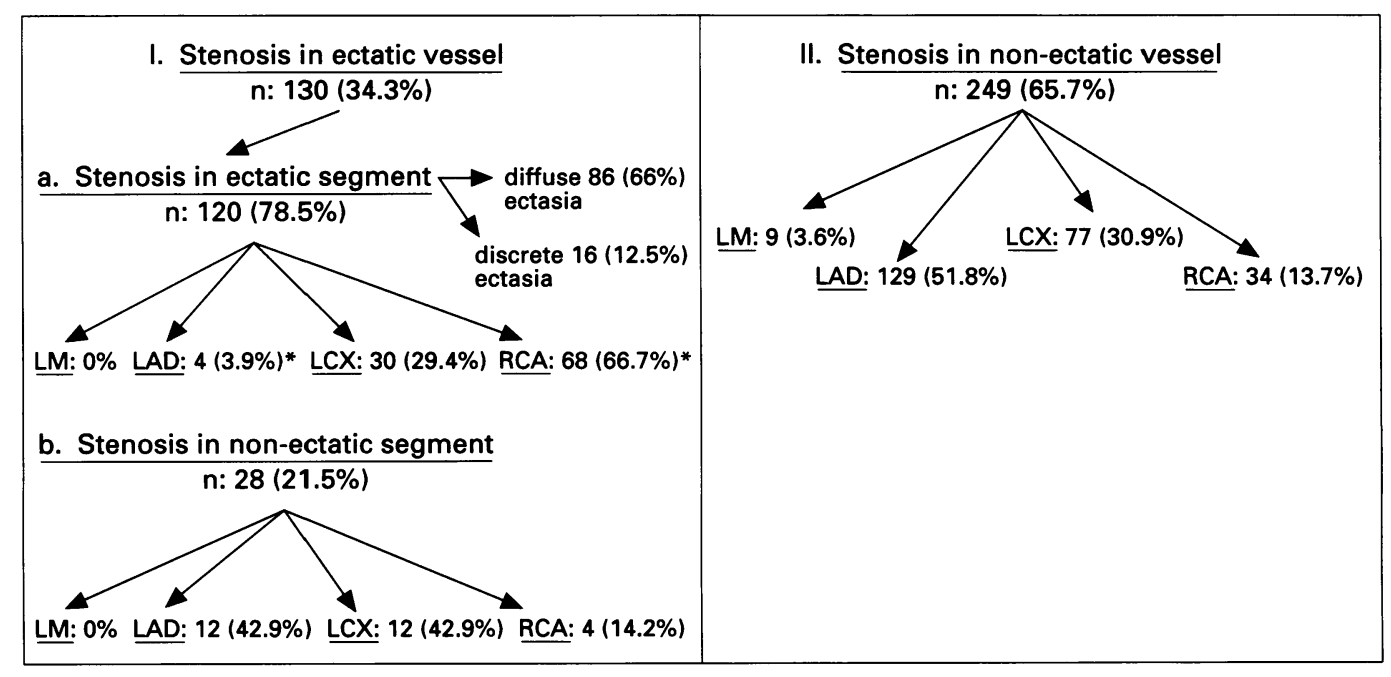


Table 2 Collateral circulation

\begin{tabular}{lll}
\hline Vessels involved & $\begin{array}{l}\text { Group } A \\
n(\%)\end{array}$ & $\begin{array}{l}\text { Group } C \\
n(\%)\end{array}$ \\
\hline 0 & $37(51 \cdot 4)$ & $39(52)$ \\
1 & $14(19 \cdot 4)$ & $9(12 \cdot 4)$ \\
2 & $7(9 \cdot 7)$ & $14(17 \cdot 8)$ \\
3 & $14(19 \cdot 4)$ & $14(17 \cdot 8)$ \\
\hline
\end{tabular}

There were no significant differences between the two groups. Group A, patients with coronary ectasia and coexisting
coronary artery disease (CAD); Group C, patients with significant $\mathrm{CAD}$ without ectasia.

ectatic segment, the right coronary artery was much more often affected, and the left anterior descending much less often $(P<0.001)$.

In group $B$, a further subgroup analysis was carried out in the 12 patients who had been diagnosed as having had a previous myocardial infarction. Of these, three had had a non-Qwave infarct. At left ventriculography none had localised hypokinesia. By electrocardiographic diagnosis, the location of the infarct could be documented reliably in two. In both of these, the ectatic artery corresponded to the infarct location; the left anterior descending artery was ectatic in an anterior and the left circumflex artery in a lateral infarction.

Nine patients had been diagnosed as having had a $Q$ wave infarct. Of these, five had wall hypo- or akinesis at ventriculography. In all nine, the artery corresponding to the infarct was ectatic, while in seven this was the only ectatic vessel; all had had an inferior infarctssix had ectasia of the right coronary and one of a dominant left circumflex artery. In two patients all three vessels were ectatic; one had an anterolateral and one an inferior infarct.

There was no difference in the collateral vessel picture between groups $A$ and $C$ (table 2).

The angiographically estimated ejection fraction was identical in groups A $(48.3 ; 10 \%)$ and $C(49.3 ; 10 \%)$ but significantly higher in group $\mathrm{B}(56.5 ; 9 \% ; \mathrm{P}<0.001)$.

\section{FOLLOW UP}

In our three groups around $70 \%$ of the patients had follow up data available. The proportion with unstable angina, acute myocardial infarction, or death was similar in groups $A$ and $C$ (table 3 ). In group $B$ no death or acute myocardial infarction occurred, and only one episode of unstable angina which was successfully managed. Because of the small numbers, untoward events were not significantly different among the three groups. More

Table 3 Follow up characteristics

\begin{tabular}{lccc}
\hline & Group $A$ & Group B & Group C \\
\hline n (\% of total) & $121(70 \cdot 3)$ & $21(68)$ & $115(69 \cdot 6)$ \\
Follow up, months (SD) & $29(14)$ & $28(13)$ & $26(10)$ \\
Medical treatment, n (\%) & $53(44)$ & $21(100)$ & $43(37 \cdot 4)$ \\
CABG, n (\%) & $47(39)$ & - & $42(36 \cdot 5)$ \\
PTCA, n (\%) & $7(5 \cdot 8)$ & - & $20(17 \cdot 4)^{\star}$ \\
Unstable angina, n (\%) & $9(7 \cdot 5)$ & - & $5(4 \cdot 4)$ \\
Myocardial infarction, n (\%) & $2(1 \cdot 6)$ & - & $1(0.9)$ \\
Cardiac death, n (\%) & $4(3 \cdot 3)$ & $15 \cdot 2)$ \\
Unstable angina, myocardial & $15(12 \cdot 4)$ & $1(4 \cdot 8)$ & $12(10 \cdot 4)$ \\
\hline infarction, cardiac death, n (\%) & 15 & \\
\hline
\end{tabular}

$\star \mathrm{P}<0.01 v$ group $\mathrm{A}$

Group A, patients with coronary ectasia and coexisting coronary artery disease (CAD); Group B patients with coronary artery ectasia without sionificant coexisting CAD; Group C, patients with significant CAD without ectasia.

CABG, coronary artery bypass graft; PTCA, percutaneous transluminal coronary angioplasty. patients in group $\mathrm{C}$ underwent percutaneous coronary angioplasty than in group $A(P<$ 0.01 ), while the number of bypass operations was the same. No patient in group B needed surgical or interventional treatment. Most of these patients were on triple anti-ischaemic treatment (nitrates, $\beta$ blockers, and calcium antagonists) and aspirin.

\section{Discussion}

During recent years numerous studies have dealt with aneurysmal coronary artery disease. In the majority the numbers of patients were modest. The largest study was carried out in the context of CASS, in which 20087 patients were studied, among whom $978(4.9 \%)$ were found to have coronary ectasia. ${ }^{1}$ In most of the previous reports the incidence has varied between $1.2 \%$ and $4.9 \%$. Only Sharma et al ${ }^{10}$ found an incidence as high as $10-12 \%$, but in an Indian population, which may have different demographic characteristics. The incidence of $5 \cdot 3 \%$ in our study is similar to that of CASS. However, the estimation of the degree of stenosis is not easy in the presence of aneurysmal disease. Furthermore, we are not aware of the use of automated measurement techniques in the presence of ectasia. Thus we employed the criteria used by the CASS researchers. Our attempt to differentiate between stenosis in an ectatic and in a nonectatic vessel or segment has not been done before to our knowledge. In group A, when the non-ectatic vessel was involved, the left anterior descending coronary artery was the vessel most frequently affected, while when the stenosis was situated in an ectatic segment, the right coronary artery was most commonly affected.

As regards the aetiology, no specific predisposing factors have been identified. Male sex has been found to predominate. ${ }^{1311}$ In our population this cannot be assessed, since our groups $A$ and $C$ were matched for age and sex. Group B had a similar male to female ratio to groups $A$ and $C$. A positive family history and hypertension have been cited. $^{2}$ Hypercholesterolaemia seems to be a predisposing factor; in a recent report familial hypercholesterolaemia was accompanied by coronary ectasia in $15 \%$ of patients, as compared with $2.5 \%$ of an age and sex matched control group. ${ }^{12}$ Aneurysmal coronary arteries have been associated with polyarteritis nodosa, ${ }^{13}$ Takayasu's disease, ${ }^{14}$ lymphadenopathy, ${ }^{15}$ trauma, ${ }^{16}$ and congenital dysplasia. ${ }^{17}$ Age seems to have no additional influence according to most investigators. Specifically, Sharma et $a l^{10}$ found no difference of ectasia in younger and older patients. A common factor in all of these conditions is the weakening of the media with a diminution of its elastic elements in areas of marked atherosclerosis ${ }^{4}$ which permits the dilatation of the vessel wall at the area of defective structure. This mechanism is probably different from that of the poststenotic dilatation, because it can be seen in vessels without any stenosis and in any segment of their whole length. Here a differentiation must 
be made between diffuse aneurysmal ectasia and discrete aneurysm formation: according to Tunick et $a l^{18}$ a discrete aneurysm is defined as a localised abnormal dilatation of the coronary artery that is spherical or saccular. These investigators specifically excluded patients with fusiform aneurysms or with a spindle shape involving a large segment of the artery; they classify such cases together with ectasia. According to them, discrete aneurysms are much less common than diffuse ectasia and they are never found in arteries without severe stenosis. The underlying disease is more severe in patients with discrete aneurysms than in those with diffuse ectasia. Discrete aneurysms are also more common in the left anterior descending coronary artery while ectatic disease is more common in the right coronary artery. Although the distribution of ectasia was different in our groups $A$ and $B$, the incidence of discrete ectasia in one vessel (type IV) was similar in the two groups.

Coronary artery disease seems to be the main underlying aetiology for coronary artery ectasia, ${ }^{19}$ since $84.7 \%$ in our study, $90.8 \%$ in CASS, ${ }^{1}$ and $82.5 \%$ in the series of Farto-eAbreu et al ${ }^{20}$ had coexisting stenotic coronary artery disease. An increased incidence of aneurysms of the abdominal aorta has been found and has been attributed to a similar histological picture in the two conditions, characterised by a destruction of the musculo-elastic elements of the tunica media due to the atherosclerotic process. ${ }^{131921} \mathrm{La}$ Mendola et $a l^{22}$ have also reported a case of multiple coronary aneurysms in a patient with an abdominal aortic aneurysm and bilateral popliteal artery aneurysms.

Our findings indicate that, with a similar number of significant obstructive coronary lesions, exercise tolerance, incidence of angina pectoris or a previous $Q$ wave or non- $Q$ wave myocardial infarct, and events at follow up are comparable in patients with coronary artery disease with and without coronary ectasia, while patients with ectasia but insignificant coronary artery disease present with a definitely more benign picture.

We believe that our study is one of few to place the problem of coronary ectasia and its association or lack of association with significant coronary artery disease in its true perspective, for the following reasons. Two previous series had too few patients with ectatic coronary arteries without significant obstructive disease to yield any significant conclusions. ${ }^{311}$ Aintablian's series ${ }^{7}$ consisted only of patients with significant coronary artery disease, while among the 17 patients of Stajduhar et $\mathrm{al}^{4}$ the majority had discrete aneurysms. In the series of Markis $e t a l^{2}$ no distinction was made between patients with coronary artery ectasia with and without coronary artery disease. Anyway, because of their small overall numbers (30) such differences would not be meaningful. Their data show that their patients had a significantly higher incidence of angina than ours, but a similar incidence of old myocardial infarction to our group A patients $(60 \% v 61 \cdot 6 \%)$, assessed by resting electrocardiogram. Their follow up results at two years were more unfavourable than ours: a $20 \%$ incidence of cardiac deaths compared to a $3.3 \%$ incidence in our group A. However, 18 years have passed between their series and ours, with great changes in the medical treatment of coronary artery disease and a resulting significant improvement in long term mortality.

The CASS study, ${ }^{1}$ the only one with larger numbers of patients than ours, arrived at essentially the same conclusions. Their comparisons were as follows:

(1) 957 patients with aneurysmal disease but with any degree of coronary stenosis below $70 \% \quad(69 / 957=7 \cdot 2 \%)$ or above $70 \%$ $(888 / 957=92 \cdot 8 \%)$ were compared with a group with a similar degree of coronary stenosis but without ectasia. In these two groups no significant differences were noted when adjustment for arteriographic and ventriculographic findings was carried out, but patients with aneurysmal disease had a higher incidence of documented myocardial infarction, in contrast to our findings.

(2) Patients with aneurysmal disease but with insignificant or no coronary artery disease (90 patients) were compared with those with no significant obstructive or aneurysmal disease. None of these control individuals had normal arteries. They found no difference between these two groups except for male preponderance, which has already been noted. In our study, patients with coronary ectasia and without significant coronary artery disease (group B) also had a lower incidence of a previous myocardial infarction, a higher exercise time and ejection fraction, and significantly better follow up results than patients with coexisting coronary artery disease (group A). Moreover, if the rates of percutaneous transluminal coronary angioplasty and aorto-coronary bypass in patients with myocardial infarction, unstable angina, and cardiac death are summed, it can be seen that those with no significant obstructive lesions had far fewer follow up events than the two groups with significant disease, regardless of the presence of ectasia. These results are similar to the findings of Farto-e-Abreu et $a l^{20}$ who followed 30 patients with ectasia, of whom seven had pure ectasia, without stenotic lesions, for $63 \cdot 1$ (SD 30.5) months. Of the nine events, one death, three myocardial infarctions, and five episodes of unstable angina, eight occurred in the group with coexisting lesions, and only one episode of unstable angina occurred in the group with pure ectasia and normal coronary arteries.

One point worth mentioning is that, with a comparable severity of obstructive coronary artery disease, patients without ectasia underwent percutaneous transluminal coronary angioplasty three times more often. It is possible that their physicians were intuitively reluctant to recommend this intervention in this group of patients. However, the published data are scant. Ochiai et $a l^{23}$ concluded that angioplasty seems an effective and safe treatment for stenoses adjacent to aneurysmal segments. 
Thus from our own and the previous investigators' results we can draw the following conclusions. (1) Patients with coronary ectasia and coronary obstructive disease are similar in every respect to those with similar coronary obstructive disease but without ectasia. (2) Pure ectasia is not completely innocuous, since there is an appreciable incidence of a previous myocardial infarction and angina (both $39 \%$ in our series). Thus the notion that slow flow may occur-even in the absence of atherosclerotic lesions and in situ thrombosis-is compatible with our findings. Moreover distal embolisation from mural thrombus within the ectatic segment cannot be excluded. It should be noted that in our series the electrocardiographic localisation of the infarct was always associated with an ectatic coronary artery. This correlation is reported for the first time. Hartnell et al, ${ }^{12}$ in contrast, do not believe that angina is more frequent in this entity. However, overall these patients have a significantly more favourable outcome than patients with obstructive disease.

Finally, our findings raise one more question with regard to the treatment of the patients with coexisting disease and with pure ectasia. Since their follow up course is favourable, the recommendation of Swanton $e t$ $\mathrm{al}^{3}$ for systemic anticoagulation instead of aspirin may need to be reconsidered. These investigators studied only one patient with pure ectasia, but they based their recommendation-which has been reiterated since then-on the conjecture that patients with coronary artery ectasia should be anticoagulated "in view of the tendency for mural thrombosis to occur in arteriomegalic leg vessels, cerebral arteries, and probably coronary arteries". This last reference concerns a case study. ${ }^{24}$ Anticoagulation carries a certain risk of haemorrhage. According to Caro et al ${ }^{25}$ the expected benefit of anticoagulation must be six times higher than the danger of haemorrhage. With no infarcts or death in two years in our series and in five years in the series of Farto-e-Abreu, ${ }^{20}$ it would be difficult to justify classically defined anticoagulant treatment, unless mini-dose anticoagulation was employed. However, very large numbers of patients would be required for such a study. Moreover, it should not be forgotten that even in large follow up studies of patients with aortocoronary vein grafts, oral anticoagulants have not proven superior to aspirin. ${ }^{26}$

In conclusion, our findings, as well as of other studies, do not suggest that aneurysmal coronary artery disease should be considered a distinct anatomical or functional abnormality. It seems that it is a variation of coronary artery disease, with which it usually coexists.
1 Swaye PS, Fisher LD, Litwon P, Vingela PA, Judkins MP, Kemp HG, et al. Aneurysmal coronary artery disease. Circulation 1983;67:134-8.

2 Markis JE, Joffe CD, Cohn PF, Feen DJ, Hermann MV Gorlin R. Clinical significance of coronary arterial ectasia. Am ₹ Cardiol 1976;37:217-22.

3 Swanton RH, Thomas MC, Coltart DJ, Jenkins BS, Webb Peploe MM, Williams BT. Coronary artery ectasiavariant of occlusive coronary arteriosclerosis. Br Heart $f$ 1978;40:393-400.

4 Stajduhar KC, Laired JR, Rogan KM, Wortham DC. Coronary arterial ectasia: increased prevalence in patients with abdominal aortic aneurysm as compared to occlusive atherosclerotic peripheral vascular disease. Am Hear Ұ 1993;125:86-92.

5 Befeler B, Aranda JM, Embi A, Mullin FL, El-Sherif N, Lazzara R. Coronary artery aneurysms. Study of their etiology, clinical course and effect on left ventricular funcology, clinical course and effect on left ventricular

6 CASS Principal Investigators and their Associates. Coronary Artery Surgery Study (CASS): a randomized Coronary Artery Surgery Study (CASS): a randomized
trial of coronary artery bypass surgery, survival data. trial of coronary artery bypas
Circulation 1983;68:939-50.

7 Aintablian A, Hamby RT, Hoffman I, Kramer RJ. Coronary ectasia: incidence and results of coronary bypass surgery. $\mathrm{Am}$ Heart $\mathcal{f}$ 1978;96:308-15.

8 Sandler H, Dodge HT. The use of single plane angiogram for the calculation of left ventricular volume in man. $A m$ Heart F 1968;75:325-34

9 Cohen M, Rentrop P. Limitation of myocardial ischemia by collateral circulation during sudden controlled coronary artery occlusion in human subjects: a prospective study. Circulation 1986;74:469-76.

10 Sharma SN, Kaul U, Sharma S, Wasiv HS, Manchanda SC, Bahl VK, et al. Coronary arteriographic profile in young and old Indian patients with ischemic heart disease: a comparation study. Indian Heart $f$ 1990;42: ease: a

11 Hartnell GG, Parnell BM, Pridie RB. Coronary artery ectasia. Its prevalence and clinical significance in 4993 patients. Br Heart $\mathcal{F}$ 1985;54:392-5.

12 Sudhir K, Ports TA, Amidon TM, Goldberger JJ, Kane JP, Yock $\mathrm{P}$, et al. Increased prevalence of coronary ectasia in heterozygous familial hypercholesterolemia. Circulation 1995;91:1375-80.

13 Holsinger DR, Osmundson PJ, Edwards JE. The heart in periarteritis nodosa. Circulation 1962;25:610-18.

14 Ellis R, Kurth RS. Calcified coronary artery aneurysm. fAMA 1968;203:51-3.

15 Kitamura S, Kawashima Y, Miyamoto K, Kobayashi T, Matsuda H, Ohgitani N, et al. Multiple coronary artery aneurysms resulting in myocardial infarction in a young man. ₹ Thorac Cardiovasc Surg 1975;70:290-7.

man. F Thorac Cardiovasc Surg 1975;70:290-7.
16 Loring WE. Multiple arterioslerotic aneurysms of coronary arteries. Arch Pathol 1955;59:449-52.

17 Anabtami IN, De Leon JA. Arteriosclerotic aneurysms of the coronary arteries. $\mathcal{F}$ Thorac Cardiovasc Surg 1974; 68:226-8.

18 Tunick PA, Slater J, Kronzon I, Glassman E. Discrete atherosclerotic coronary artery aneurysm: a study of 20 patients. F Am Coll Cardiol 1990;15:279-82.

19 Starc R, Janezic AS, Cijan A. Angiographic evidence of coronary artery ectasia: our experience. Cor Vasa 1991; 33:288-93.

20 Farto-e-Abreu P, Mesquita A, Silva JA, Seabra Comez R. Coronary artery ectasia: clinical and angiographic characteristics and prognosis. Rev Port Cardiol 1993;12:305-10.

21 Daoud AS, Pankin D, Tulgan H, Florentin RA. Aneurysms of the coronary artery-report of ten cases and review of literature. Am f Cardiol 1963;11:228-37.

$22 \mathrm{La}$ Mendola CL, Culliford AT, Harris LJ, Amendo MT Multiple aneurysms of the coronary arteries in a patient with systemic aneurysmal disease. Am $\mathcal{f}$ Thorac Surg with systemic ane

23 Ochiai M, Yamaguchi T, Taguchi J, Ohno M, Yoshimura $\mathrm{H}$, Kashida $\mathrm{M}$, et al. Angioplasty of stenoses adjacent to aneurysmal coronary artery disease. $\mathcal{F p n}$ Heart $\mathcal{F} 1990 ; 31$ : 749-57.

24 Ebert PA, Peter RH, Gunnells JC, Sabiston DC. Resecting and grafting of coronary artery aneurysm. Circulation 1971;43:593-8.

25 Caro J, Croome PA, Flegel KM. Atrial fibrillation and anticoagulation: from randomised trials to practice. Lancet 1993;341:1381-4.

26 Van der Meer J, De la Riviere AB, Van Gilst WH, Hillege HL, Pfisterer M, Kootstra GJ, et al. Effects of low dose aspirin $(50 \mathrm{mg} /$ day), low dose aspirin plus dipyridamole and oral anticoagulant agents after internal mammary and oral anticoagulant agents after internal mammary year. $\mathcal{F}$ Am Coll Cardiol 1994;24:1181-8. 